\title{
New Strategy on Materials Paradigm \\ for Highly Efficient Dye-Sensitized Solar Cells
}

\author{
Hwan Kyu Kim \\ Department of Advanced Materials Chemistry Korea University (Sejong), ChungNam 339-700 Korea \\ Email: hkk777@korea.ac.kr
}

\begin{abstract}
Dye-sensitized solar cells (DSSCs) have attracted considerable interest because of their higher conversion efficiency of sunlight-to-electricity and potential low costs of production and materials. On the basis of systematic device engineering benefited from continuous efforts on material innovation and device physics, a state of the art DSC with a highly volatile electrolyte has achieved a validated efficiency of $11.1 \%$. Despite the high conversion efficiencies obtained for the DSSCs with liquid electrolytes, potential problems caused by liquid electrolytes, such as leakage and volatilization of the liquid, possible desorption and photodegradation of the attached dyes, and the corrosion of the counter electrode, have brought about difficulties in cell fabrication and limited the long-term performance and practical use of these DSSCs. Considering a recent gradual increment in the highest cell performance of quasi- or solid-state dye-sensitized solar cells to solve potential problems with liquid electrolytes, it is of utmost importance to reconsider the basic principle of dye-sensitized solar cells for the remarkable breakthrough. Elucidation of the relationship between molecular structure, film structure, device photophysics, photovoltaic properties of dyes and co-adsorbents as well as polymeric electrolytes would be a prerequisite to overcome the situation. Here, new strategy on materials paradigm for highly efficient dye-sensitized solar cells will be described.
\end{abstract}

\section{References}

1. N. S. Baek and H. K. Kim, et.al., Energy and Environmental Science, 2, 1082 (2009).

2. K. D. Seo and H. K. Kim, et. al., Dyes and Pigments, 90, 304 (2011).

3. B. J. Song, I. T. Choi, M. J. Ju and H. K. Kim, et.al., Chem. Eur. J., 17, 11115 (2011).

4. H. M. Song, I. T. Choi and H. K. Kim, et. al., J. Mater. Chem., 22, 3786 (2012).

5. M. S. Kang, S. H. Kang and H. K. Kim, et. al., Chem. Comm. DOI:10.1039/c2cc31384g (2012.04). 